\title{
PACKET SCHEDULING OF STREAMING VIDEO WITH FLEXIBLE REFERENCE FRAME USING DYNAMIC PROGRAMMING AND INTEGER ROUNDING
}

\author{
Gene Cheung, Wai-tian Tan
}

Hewlett-Packard Laboratories

\begin{abstract}
Video coding standards like H.264 offer the flexibility to select reference frames during motion estimation for predicted frames. We investigate the packet scheduling problem of streaming video over lossy networks from a real-time encoder with flexible reference frame. In particular, we consider a multi-path streaming setting where each predicted frame of video, in addition to the flexibility to select a reference frame, can schedule one or multiple transmissions on one or multiple delivery paths for the upcoming optimization period. We present an algorithm based on dynamic programming that provides a locally optimal solution with high complexity. We then present a rounding method to reduce computation complexity at the expense of degrading solution quality. Results show that our algorithm performs noticeably better than a greedy scheme, and graceful tradeoff between complexity and solution quality can be achieved.
\end{abstract}

\section{INTRODUCTION}

Advances in video coding and networking have created new flexibilities in the design of streaming algorithms. An example is reference frame selection (RFS) of video coding standards like H.264 [1], where each coding block within a predicted frame can choose among several previously encoded frames for motion prediction. This allows a live encoder to avoid using lost frames as references, thereby controlling error propagation. Another example is multi-homing, where a client is equipped with multiple network interfaces, such as $802.11 \mathrm{~b}$ and WCDMA. Using both interfaces has obvious advantages including higher throughput and less variability.

While it is clear that streaming can be enhanced by exploiting these flexibilities, high complexity is required to jointly select optimal parameters for options afforded by the multiple flexibilities. Specifically, based on feedbacks, at a given optimization instance a live encoder must choose reference frames for predicted frames and transmit frames one or multiple times using one or multiple interfaces. To make such selection, we investigate an algorithm based on dynamic programming that produces a locally optimal solution with high complexity. We then apply an integer rounding technique to the algorithm to reduce the complexity at the expense of degrading solution quality. Results show that our proposed algorithm performs noticeably better than a greedy algorithm derived from the simplified version of $\mathrm{RaDiO}$ [2].

The paper is organized as follows. After discussing related work in Section 2, we present our source and network models in Section 3. We present our optimization algorithm in Section 4. In Section 5, we discuss an integer roundingbased procedure to reduce the algorithm complexity at the cost of solution quality. Results and conclusion are presented in Section 6 and 7, respectively.

\section{PREVIOUS WORK}

Video coding standard H.264 [1] has superior coding performance over previous standards like MPEG-4 and H.263 over a broad range of bit rates. Part of H.264 definition is the flexibility of using any arbitrary frame to perform motionestimation, originally introduced as Annex N in H.263+ and later as Annex U in H.263++. Early work on enhancing streaming using RFS includes [3, 4]. Our work differs from these works by employing a complexity-scalable optimization procedure and also applying optimization to jointly perform reference frame (RF) and transmission path (TP) selection.

Recently, [5] proposes the reorganization of the prediction structure of a group of pictures (GOP) to minimize the effects of losing a single P-frame. We differ from [5] in two regards: i) we maximize the expected performance - the average case, while [5] minimizes the worst case; and, ii) we adapt our scheme according to observed network conditions.

Our recent work [6] has shown that dynamic programming plus integer rounding can be applied to the joint selection of reference frame, QoS and delivery path in a multi-path environment without feedbacks. This paper is a generalization in three important regards: i) we permit retransmissions of lost packets given available client feedbacks; ii) GOP structure is generalized so that any frame can be real-time encoded as an I-frame or a P-frame; and, iii) transmission delay in each path is modeled as a shifted-Gamma-distributed random variable. With these enhancements, we obtain a more general framework for packet scheduling over multiple paths with feedbacks and flexible reference frames.

\section{ASSUMPTIONS AND PROBLEM FORMULATION}

In our intended application scenario, a sender is jointly optimizing encoding of video and its transport over two network paths via two network interfaces to the client. On the live-encoder side, we choose previously encoded frames to be used for references. On the transport side, we choose the number of transmissions for each encoded frame over the two 


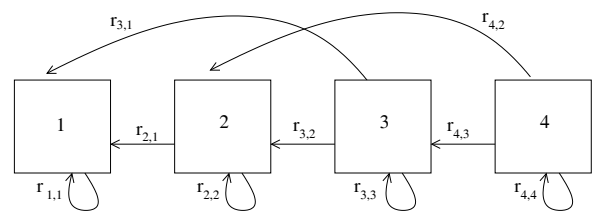

Fig. 1. Example of DAG Source Model

interfaces. We first present the source and network models, then discuss our objective function.

\subsection{Directed Acyclic Graph Source Model}

Our optimization is run periodically with period $P$, where during each optimization instance, a window of $M$ consecutive frames of a $M_{\max }$-frame video sequence is under consideration for (re)transmission, and each frame in the window can be coded either as an intra-coded frame (I-frame) or an inter-coded frames (P-frames). For simplicity, we assume frames 1 to $M$ are being optimized, though in reality they can be any $M$ consecutive frames in the $M_{\max }$-frame sequence. Each frame i $\left(F_{i}\right)$ must be delivered to the client by a playback deadline $D_{i}$ or is rendered useless. At the next optimization instance, the window advances $k$ frames in the sequence, where $k$ is the number of leading frames with playback deadlines having expired at the client. Each $F_{i}$ has a transmission history $\mathbf{h}_{i}$ which records the times and numbers of transmissions on each path the optimization had selected for $F_{i}$ in previous optimization instances (more in Section 3.2).

We model the decoding dependencies of the $M$ frames in the window using a directed acyclic graph (DAG) $G=(\mathcal{V}, \mathcal{E})$ with vertex set $\mathcal{V},|\mathcal{V}|=M$, and edge set $\mathcal{E}$, similar to [2]. Specifically, each frame $F_{i}, i \in\{1, \ldots, M\}$, represented by a node $i \in \mathcal{V}$, has a set of outgoing edges $e_{i, j} \in \mathcal{E}$ to nodes $j$ 's. $F_{i}$ can use $F_{j}$ as reference iff $\exists e_{i, j} \in \mathcal{E}$. We define $x_{i, j}$ to be the binary variable indicating whether $F_{i}$ uses $F_{j}$ as reference. Equivalently, given $i$, we define $x_{i, j}$ as:

$$
x_{i, j}= \begin{cases}1 & \text { if } F_{i} \text { uses } F_{j} \text { as RF } \quad \forall j \in \mathcal{V} \mid e_{i, j} \in \mathcal{E} \\ 0 & \text { otherwise }\end{cases}
$$

H.264 syntax generally allows different coding blocks to use different reference frames. Instead, we restrict all blocks in a frame to use the same reference frame to reduce optimization complexity. We now have the following $R F$ constraint:

$$
\sum_{\forall j \mid e_{i, j} \in \mathcal{E}} x_{i, j}=1 \quad \forall i \in \mathcal{V} \mid i \neq 1
$$

We assume that only frames in the past are used for reference, i.e. $\forall e_{i, j} \in \mathcal{E}, i \geq j$. Further, we limit the number of candidate reference frames for any predicted frame $F_{i}$ to be $E_{\text {max }} \ll M$. An example of a DAG of a 4 -frame sequence is shown in Figure 1 with $E_{\max }=2$. We denote by $r_{i, j}$ the bit count of $F_{i}$ when $F_{j}$ is used as reference. We assume a sparse rate matrix $\mathbf{r}$ of size $\mathcal{O}\left(M^{2}\right)$ is computed a priori (to be discussed in Section 6) as input to the optimization algorithm (sparse because each row has at most $E_{\max }$ entries).

\subsection{Network Model}

We first assume that the network imposes a maximum transport unit of size $M T U$ bytes, so that frame of size larger than $M T U$ will be fragmented. For transmission of packet of size $\leq M T U$ bytes on path $k$, we assume a time-invariant packet erasure channel with random delay as in [2]. More specifically, let $\pi_{k}$ be the packet erasure probability of path $k$, and $g_{k}(\gamma)$ be the shifted Gamma distribution with parameters $\alpha_{k}$, $\lambda_{k}$ and $\kappa_{k}$ that describes the probability distribution of delay random variable $\gamma_{k}$ of path $k$ :

$$
g_{k}\left(\gamma_{k}\right)=\frac{\lambda_{k}^{\alpha_{k}}\left(\gamma_{k}-\kappa_{k}\right)^{\alpha_{k}-1} e^{-\lambda_{k}\left(\gamma_{k}-\kappa_{k}\right)}}{\Gamma\left(\alpha_{k}\right)} \quad \kappa_{k}<\gamma_{k}<\infty
$$

where $\Gamma\left(\alpha_{k}\right)$ is the Gamma function. This means that a packet sent on path $k$ at time $t_{o}$ will have probability of correct transmission by time $T, \delta_{k}(t), t=T-t_{o}$, defined by:

$$
\delta_{k}(t)=\left(1-\pi_{k}\right) \int_{\kappa_{k}}^{t} g_{k}(\gamma) d \gamma
$$

We allow each $F_{i}$ to select two transmission levels (QoS) $q 0_{i}, q 1_{i} \in \mathcal{Q}=\{0,1, \ldots Q\}$ indicating the number of (re)transmissions until the next optimization instance on path 0 and 1 , respectively. At a given optimization instance $t_{o}$, selection of QoS level $q 0_{i}$ and $q 1_{i}$ and frame size $r_{i, j}$, together with $F_{i}$ 's transmission history $\mathbf{h}_{i}$, will induce a frame delivery success probability $p\left(\mathbf{h}_{i}, q 0_{i}, q 1_{i}, r_{i, j}\right) \in \mathcal{R}$.

We derive $p\left(\mathbf{h}_{i}, q 0_{i}, q 1_{i}, r_{i, j}\right)$ given our network model. We first define $F_{i}$ 's history $\mathbf{h}_{i}$ of length $l_{i}$ as:

$$
\begin{aligned}
\mathbf{h}_{i}= & \left\{\left(f_{i}, q 0_{i}^{(1)}, q 1_{i}^{(1)}, t_{i}^{(1)}\right),\left(q 0_{i}^{(2)}, q 1_{i}^{(2)}, t_{i}^{(2)}\right), \ldots,\right. \\
& \left.\left(q 0_{i}^{\left(l_{i}\right)}, q 1_{i}^{\left(l_{i}\right)}, t_{i}^{\left(l_{i}\right)}\right)\right\}
\end{aligned}
$$

where the reference frame $F_{j}$ selected for $F_{i}$ is denoted by $f_{i}$, and QoS selections and transmission time of instance $k$ are denoted by $q 0_{i}^{(k)}, q 0_{i}^{(k)}$ and $t_{i}^{(k)}$, respectively. Let $n_{i}=$ $\left\lceil\frac{r_{i, f_{i}}}{8 * M T U}\right\rceil$ be the number of packets required to encode $F_{i}$ using reference frame $f_{i}$. The frame delivery failure probability of using QoS $q 0_{i}$ at time $t_{o}$ is then $\zeta_{0}\left(q 0_{i}, n_{i}, D_{i}-t_{0}\right)$ :

$$
\zeta_{0}\left(q 0_{i}, n_{i}, D_{i}-t_{0}\right)=\left(1-\delta_{0}\left(D_{i}-t_{0}\right)^{n_{i}}\right)^{q 0_{i}}
$$

If $F_{i}$ is not ACKed, $p\left(\mathbf{h}_{i}, q 0_{i}, q 1_{i}, r_{i, j}\right)$ can be written as:

$$
\begin{array}{r}
p\left(\mathbf{h}_{i}, q 0_{i}, q 1_{i}, r_{i, j}\right)=1-\zeta_{0}\left(q 0_{i}, n_{i}, D_{i}-t_{o}\right) \zeta_{1}\left(q 1_{i}, n_{i}, D_{i}-t_{o}\right) \\
\prod_{k=1}^{l_{i}} \zeta_{0}\left(q 0_{i}^{(k)}, n_{i}, D_{i}-t_{i}^{(k)}\right) \zeta_{1}\left(q 1_{i}^{(k)}, n_{i}, D_{i}-t_{i}^{(k)}\right)
\end{array}
$$

$p\left(\mathbf{h}_{i}, q 0_{i}, q 1_{i}, r_{i, j}\right)=1$ if $F_{i}$ is ACKed.

\subsubsection{Network Resource Constraint}

We impose network constraints on the amount of resource we can use per optimization period. Accordingly, the constraints for path 0 and path 1 are respectively:

$$
\begin{aligned}
\sum_{i=1}^{M} \sum_{\forall j \mid e_{i, j} \in \mathcal{E}} x_{i, j} q 0_{i} r_{i, j} \leq \bar{R}_{0} \\
\sum_{i=1}^{M} \sum_{\forall j \mid e_{i, j} \in \mathcal{E}} x_{i, j} q 1_{i} r_{i, j} \leq \bar{R}_{1}
\end{aligned}
$$


Constraint parameters $\bar{R}_{0}$ and $\bar{R}_{1}$ are network available bandwidth scaled by optimization period $P$. Network bandwidth can be estimated, for example, using congestion control algorithms like TCP-friendly rate control (TFRC).

\subsection{Objective Function}

The objective function we selected is the expected number of correctly decoded frames at the decoder for the $M$ frames in the optimization window. Each frame $F_{i}$ is correctly decoded iff $F_{i}$ and all frames $F_{j}$ 's it depends on are delivered on-time and drop-free. We write $j \preceq i$ if frame $F_{i}$ depends on frame $F_{j}$. Mathematically, maximizing this objective function means computing:

$\max _{\left\{x_{i, j}\right\},\left\{q 0_{i}\right\},\left\{q 1_{i}\right\}}\left\{\sum_{i=1}^{M} \prod_{\forall j \preceq i} \sum_{\forall k \mid e_{j, k} \in \mathcal{E}} x_{j, k} p\left(\mathbf{h}_{j}, q 0_{j}, q 1_{j}, r_{j, k}\right)\right\}$

The problem is then: given pre-computed rate matrix $\mathbf{r}$ and delivery success probability function $p\left(\mathbf{h}_{i}, q 0_{i}, q 1_{i}, r_{i, j}\right)$, find variables $\left\{x_{i, j}\right\},\left\{q 0_{i}\right\}$ and $\left\{q 1_{i}\right\}$ that maximize (9) while satisfying the RF constraint (2) and the network resource constraints (8). This formally defined optimization is called the RF / QoS / Path scheduling problem (RQP scheduling).

\section{DYNAMIC PROGRAMMING ALGORITHM}

RQP scheduling can be easily shown to be NP-hard. To tackle the problem, we first construct a weakly exponential algorithm using dynamic programming to produce a locally optimal solution. Second, we introduce a rounding technique that allows multiple complexity-quality tradeoff points.

The algorithm composes of two recursive functions: Sum() and $\operatorname{Prod}()$. Sum $\left(i, R_{0}, R_{1}\right)$ returns the locally optimal expected number of correctly decodable frames for frame $F_{1}$ to $F_{i}$ given available network resources $R_{0}$ and $R_{1}$ for path 0 and 1. $\operatorname{Prod}\left(j, i, R_{0}, R_{1}\right)$ returns the probability that $F_{j}$ is $d e$ coded correctly given $R_{0}$ and $R_{1}$ for path 0 and 1 are locally optimally distributed from $F_{1}$ to $F_{i}$. A call to $\operatorname{Sum}\left(M, \bar{R}_{0}, \bar{R}_{1}\right)$ yields the locally optimal solution. $\operatorname{Sum}\left(i, R_{0}, R_{1}\right)$ and $\operatorname{Prod}\left(j, i, R_{0}, R_{1}\right)$ are shown in Figure 2 and 3 , respectively.

The recursive case (line 5-15) of $\operatorname{Sum}\left(i, R_{0}, R_{1}\right)$ locally tests every combination of RF $j$ and QoS $q 0$ and $q 1$ for $F_{i}$ for the maximal expected number of decodable frames. For a given selection of RF $j$ and QoS $q 0$ and $q 1$, it induces a resource expenditure of $q 0 r_{i, j}$ and $q 1 r_{i, j}$ for path 0 and 1 respectively, and hence a decoding probability for $F_{i}$ of $p\left(\mathbf{h}_{i}, q 0, q 1, r_{i, j}\right) * \operatorname{Prod}\left(j, i-1, R_{0}-q 0 r_{i, j}, R_{1}-q 1 r_{i, j}\right)$. That is added to the expected sum for $F_{1}$ to $F_{i-1}$ - the recursive term $\operatorname{Sum}\left(i-1, R_{0}-q 0 r_{i, j}, R_{1}-q 1 r_{i, j}\right)$.

The results of the local search are stored in the $\left[i, R_{0}, R_{1}\right]$ entries of the four dynamic programming (DP) tables (line 16-17). DP tables are lookup tables so that if the same subproblem is called again, the already computed result can be simply looked up and returned (line 1-2).

The complexity of $\operatorname{Sum}\left(M, \bar{R}_{0}, \bar{R}_{1}\right)$ is bounded by the time required to populate the DP tables, which can be easily shown to be $\mathcal{O}\left(M E_{\max } Q^{2} \bar{R}_{0} \bar{R}_{1}\right)$.

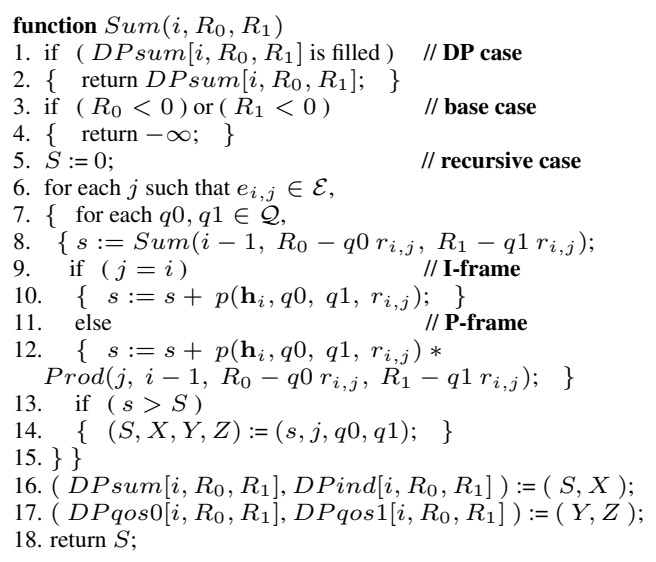

Fig. 2. Locally Optimal Algorithm $\operatorname{Sum}\left(i, R_{0}, R_{1}\right)$

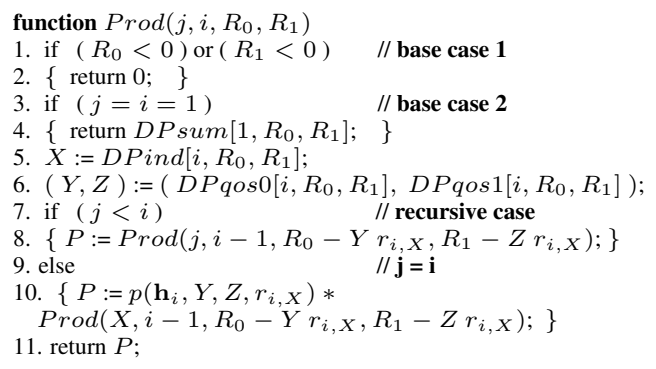

Fig. 3. Companion Function $\operatorname{Prod}\left(j, i, R_{0}, R_{1}\right)$

4.1. Companion Recursive Function $\operatorname{Prod}\left(j, i, R_{0}, R_{1}\right)$ From line 12 of Figure 2, we assume that $\operatorname{Prod}\left(j, i, R_{0}, R_{1}\right)$ is called after $\operatorname{Sum}\left(i, R_{0}, R_{1}\right)$ has been called, so we will assume entries $\left[i, R_{0}, R_{1}\right]$ of the DP tables are available during execution of $\operatorname{Prod}\left(j, i, R_{0}, R_{1}\right)$.

The recursive case has two sub-cases: i) when $j<i$ (line 8), we recurse on $\operatorname{Prod}(j, i-1,$.$) given we know resources$ $Y r_{i, X}$ and $Z r_{i, X}$ on path 0 and 1 are optimally used for node $i$; and, ii) when $j=i$ (line 10), we know term $i$ of the product term $-p\left(\mathbf{h}_{i}, Y, Z, r_{i, X}\right)$. The maximum product will be this term times the recursive term $\operatorname{Prod}\left(Y, i-1, R_{0}-\right.$ $\left.Y r_{i, X}, R_{1}-Z r_{i, X}\right)$.

\section{ROUNDING-BASED COMPLEXITY SCALING}

Having the dynamic-programming based, weakly exponential $\operatorname{Sum}\left(i, R_{0}, R_{1}\right)$, we now perform integer rounding to trade off complexity with solution quality. The rounding technique $D P$ dimension rounding [6] reduces the size of the DP tables, hence reduces complexity.

We first scale and round down budgets $\bar{R}_{0}$ and $\bar{R}_{1}$ by factor $K_{D R} \in \mathcal{R}$ - i.e. $\left\lfloor\frac{\bar{R}_{0}}{K_{D R}}\right\rfloor$ and $\left\lfloor\frac{\bar{R}_{1}}{K_{D R}}\right\rfloor$ - as input to the optimization. We then scale and round up costs of transmitting predicted frame $F_{i}, q_{i} r_{i, j}$ 's, by the same factor $K_{D R}$ - i.e. $\left\lceil\frac{q_{i} r_{i, j}}{K_{D R}}\right\rceil$. Implementationally, we accordingly rewrite line 8 and 12 of $\operatorname{Sum}\left(i, R_{0}, R_{1}\right)$ of Figure 2:

$$
\text { 8. } s:=\operatorname{Sum}\left(i-1, R_{0}-\left\lceil\frac{q 0 r_{i, j}}{K_{D}}\right\rceil, R_{1}-\left\lceil\frac{q 1 r_{i, j}}{K_{D R}}\right\rceil\right) \text {; }
$$




$$
\begin{aligned}
& \text { 12. } s:=s+p\left(\mathbf{h}_{i}, q 0, q 1, r_{i, j}\right) * \\
& \operatorname{Prod}\left(j, i-1, R_{0}-\left\lceil\frac{q 0 r_{i, j}}{K_{D R}}\right\rceil, R_{1}-\left\lceil\frac{q 1 r_{i, j}}{K_{D R}}\right\rceil\right) ;
\end{aligned}
$$

Cost terms in line 8 and 10 of $\operatorname{Prod}\left(j, i, R_{0}, R_{1}\right)$ in Figure 3 are similarly modified. Scaling down $\bar{R}_{0}$ and $\bar{R}_{1}$ means scaling down the dimension of the DP tables, hence the complexity is reduced by a factor of $K_{D R}^{2}$ at the cost of decreasing solution quality. Using $\operatorname{Sum}\left(i, R_{0}, R_{1}\right)$ and $\operatorname{Prod}\left(j, i, R_{0}, R_{1}\right)$ with the rewritten lines, the complexity of algorithm is now: $\mathcal{O}\left(M E_{\max } Q^{2} \bar{R}_{0} \bar{R}_{1} K_{D R}^{-2}\right)$.

\section{EXPERIMENTATION}

We developed a network simulator called multiple-path network simulator (muns). Each transmission path $k$ is implemented as a queue of constant service rate $\mu_{k}$, followed by an identical and independently distributed (iid) packet erasure channel with shifted-Gamma-distributed delay. Upon each packet arrival, client informs the server of its status using ACK with zero client feedback delay. Parameters for the delay distributions for path 0 and 1 are $\left(\alpha_{0}, \lambda_{0}, \kappa_{0}\right)=(3$, $0.1,80)$ and $\left(\alpha_{1}, \lambda_{1}, \kappa_{1}\right)=(2,0.1,80)$, respectively. Packet loss rates are set at $\left(\pi_{0}, \pi_{1}\right)=(0.06,0.10)$ for trial 1 , and $\left(\pi_{0}, \pi_{1}\right)=(0.04,0.08)$ for trial 2 .

We use H.264 version JM8.4 [7] to encode a 300-frame QCIF MPEG sequence news sub-sampled in time by 2, at quantization 25 and 20 for I-frames and P-frames respectively, resulting in coding rate $140.38 k b p s$ if each P-frames $F_{i}$ is coded using its previous frame $F_{i-1}$. To get rates $r_{i, j}$ 's, we iteratively force each frame $F_{i}$ to use reference $F_{i-t}$ for motion prediction during iteration $t=\{0,1,2,3,4,5\}$. The resulting coding rate is $r_{i, i-t}$. We assume $E_{\max }=5$. The optimization period is $P=300 \mathrm{~ms}$, and window size is $M=8$.

\subsection{Results 1: RQP Scheduling Comparison}

For the first set of experiment, we show that our optimization has practical merits and out-performs a competing greedy scheme. We first fixed the combined bandwidth of the two paths, $\bar{R}_{0}+\bar{R}_{1}$, at $150 \mathrm{kps}$. Rounding parameter $K_{D R}$ was kept constant at 1000 . By varying the share of $150 \mathrm{kbps}$ bandwidth to the first path bandwidth $\bar{R}_{0}$, we tracked the corresponding performance at the client in PSNR, where if a frame is not correctly decoded, the correctly decoded frame closest in the past is used to replace it. The sequence was replayed 300 times for an averaging effect.

We compared our algorithm opt to a scheme greedy that contains elements of the simplified RaDiO [2]. Specifically, given budgets $\bar{R}_{0}$ and $\bar{R}_{1}$ in the two paths, it incrementally selects the best combination of reference frame, QoS and delivery path(s) that maximizes the benefit-to-cost ratio, where benefit is the increase in objective value (9), and cost is the increase in bit expenditure. greedy proceeds until both budgets are expended.

PSNR versus path 0 bandwidth $\bar{R}_{0}$ for both schemes under both trials are shown in Figure 4a. We see that PSNR increased with $\bar{R}_{0}$. This is expected since path 0 has lower

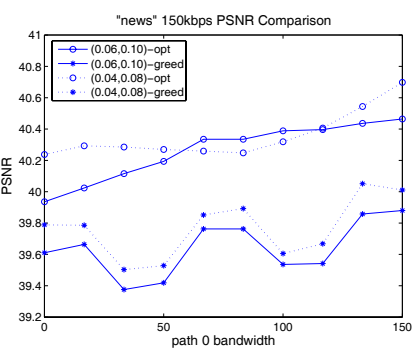

a) PSNR vs. path 0 bandwidth

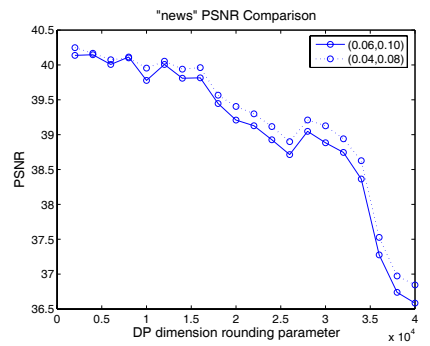

b) PSNR vs. rounding parameter
Fig. 4. Streaming Performance for news

loss rate. Further, we see that opt outperformed greedy by $0.32 d B$ to $0.85 d B$ in the trial 1 and $0.36 d B$ to $0.78 d B$ in trial 2. This provides evidence that opt is an effective scheme for RQP scheduling.

\subsection{Results 2: Performance / Complexity Tradeoff}

We next show that graceful tradeoff of performance and complexity can be achieved. We held bandwidths of the two paths $\bar{R}_{0}$ and $\bar{R}_{1}$ constant at $(50 \mathrm{kps}, 100 \mathrm{kps}) . K_{D R}$ was varied to observe the tradeoff between performance and complexity. PSNR versus $K_{D R}$ for both trials are shown in Figure 4b.

We see that as $K_{D R}$ increased, solution quality suffered due to rounding and PSNR decreased for both trials. More importantly, we see that PSNR decreased gradually over a wide range of rounding parameters. This suggests that a very wide range of useful complexity scaling can be realized using the rounding parameter $K_{D R}$.

\section{CONCLUSION}

We study packet scheduling of streaming video with flexible reference frames in a multi-path network scenario with feedbacks. In particular, we first presented an algorithm based on dynamic programming with high complexity. We then presented a rounding technique to allow multiple complexity / quality tradeoff points. Results showed that graceful complexity / quality tradeoff can be achieved over a wide range.

\section{REFERENCES}

[1] T. Wiegand, G. Sullivan, G. Bjontegaard, and A. Luthra, "Overview of the h.264/avc video coding standard," in IEEE Transactions on Circuits and Systems for Video Technology, July 2003, vol. 13, no.7, pp. 560-576.

[2] P. Chou and Z. Miao, "Rate-distortion optimized streaming of packetized media," Tech. Rep. MSR-TR-2001-35, Microsoft Research, February 2001.

[3] T. Wiegand, N. Farber, and B. Girod, "Error-resilient video transmission using long-term memory motion-compensated prediction," in IEEE J. Select. Areas. Comm., June 2000, vol. 18, no.6, pp. 1050-1062.

[4] Y.J. Liang, M. Flieri, and B. Girod, "Low-latency video transmission over lossy packet networks using rate-distortion optimized reference picture selection," in IEEE International Conference on Image Processing, Rochester, NY, September 2002.

[5] C.-M. Huang, K.-C. Yang, and J.-S. Wang, "Error resilience supporting bidirectional frame recovery for video streaming," in IEEE International Conference on Image Processing, Singapore, October 2004.

[6] G. Cheung and W. t. Tan, "Reference frame optimization for multi-path video streaming using complexity scaling," in International Packet Video Workshop, Irvine, CA, December 2004.

[7] “The TML project web-page and archive," http://kbc.cs.tu-berlin.de/ stewe/vceg/. 